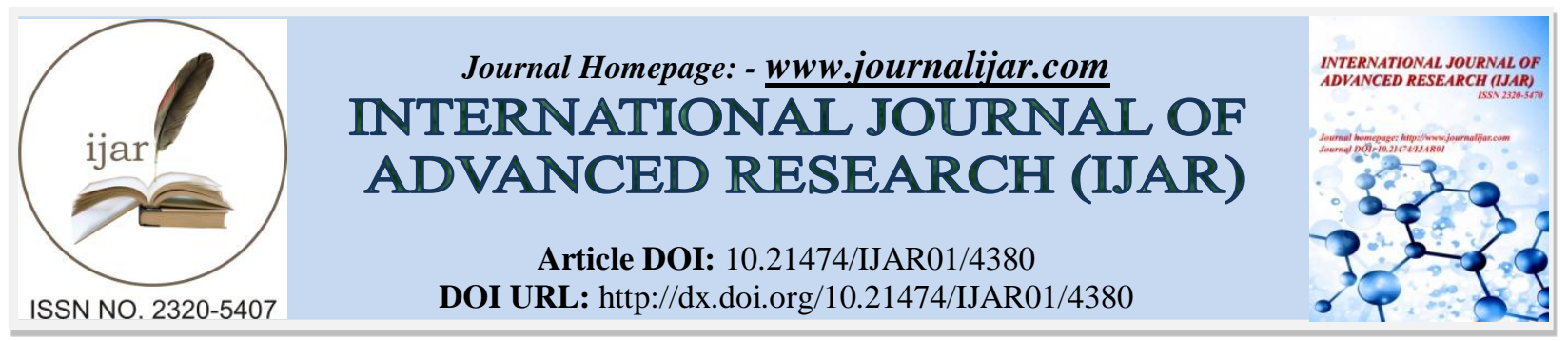

RESEARCH ARTICLE

\title{
PREDICTORS OF ATRIAL FIBRILLATION RECURRENCE AFTER CRYOBALLOON-BASED PULMONARY VEIN ISOLATION.
}

\author{
Mohamed Sanhoury MD ${ }^{1.2}$, Tarek Elzawawy MD PhD ${ }^{1}$, Mohamed Ayman Abdel-Hay MD PhD ${ }^{1}$, Mostafa \\ Nawar MD PhD ${ }^{1}$ and Antonio Curnis $\mathrm{MD} \mathrm{PhD}^{2}$. \\ 1. Cardiology and Angiology department, university of Alexandria, Egypt. \\ 2. Laboratorio di Elettrofisiologia ed Elettrostimolazione, Universita` degli Studi, Brescia, Italy.
}

\section{Manuscript Info}

Manuscript History

Received: 02 april 2017

Final Accepted: 04 june 2017

Published: june 2017

Key words:-

Atrial Fibrillation ; Pulmonary veins;

Cryoballoon; Recurrence.

\section{Abstract}

The study was designed to explore the role of imaging and clinical parameters as predictors of atrial fibrillation recurrence at 1 year after cryoballoon ablation. We included 70 patients who underwent their first cryoballoon ablation with available pre-procedural cardiac MRI study. We divided them into two groups according to AF recurrence at one year. Group A included 14 patients who developed AF recurrence at one year, while group $\mathrm{B}$ included 56 patients who remained arrhythmia-free at one year. Univariate analysis revealed a significant difference between both groups in ; age $(66 \pm 10$ years and $55 \pm 10$ years, for group A and B respectively, $p$ 0.05), left atrial diameter $(47.8 \pm 6.6 \mathrm{~mm}$ and $40.5 \pm 5.6 \mathrm{~mm}$, for group A and B respectively, $p \quad 0.001$ ), hypertension ( $71.4 \%$ and $28.6 \%$ of group A and $\mathrm{B}$ patients respectively, $\mathrm{p}$ 0.03), persistent $\mathrm{AF}(50.0 \%$ and $19.6 \%$ of group A and B patients respectively, $p \quad 0.05)$ and ERAF (57.1\% and $12.5 \%$ of group A and B patients respectively, $p$ 0.001). In subsequent multivariate regression analysis, the most significant predictor for recurrence is age. The odds of recurrence increased by $20 \%$ per one year increase in age. Moreover, one unit increase in left atrial diameter increased the chance of recurrence by $13 \%$. Conclusion; We concluded that age and left atrial diameter are independent predictors for $\mathrm{AF}$ recurrence after cryoballoon ablation. We highlight the importance of proper assessment of the risk of arrhythmia recurrence before referring the patient for ablation.

Copy Right, IJAR, 2017,. All rights reserved.

\section{Introduction:-}

$\mathrm{AF}$ is a common cardiac rhythm disturbance that increases in prevalence with advancing age. Approximately $1 \%$ of patients with AF are $<60$ years of age, whereas up to $12 \%$ of patients with AF are 75 to 84 years of age. ${ }^{(1)}$ More than one third of patients with $\mathrm{AF}$ are $>80$ years of age. ${ }^{(2,3)}$ Since its introduction into clinical practice in the late ninenties, ${ }^{(4)}$ catheter ablation of atrial fibrillation (AF) has become a well-established therapeutic option for the treatment of patients with symptomatic drug refractory AF. 
Pulmonary venous isolation (PVI) remains the cornerstone of most AF ablation procedures ${ }^{(5)}$ since PV foci were discovered as the trigger for AF. ${ }^{(6)}$ Variety of energy modalities (most commonly radiofrequency and cryoenergy ) have been used nowadays for AF catheter ablation. Point-by-point ablation using radiofrequency current is technically complex, demanding and dependent on electroanatomical mapping system (EAM) reconstruction of the left atrium and pulmonary veins ${ }^{(7)}$.Balloon-based cryoenergy creates a complete circumferential lesion around the pulmonary veins (PV) with a limited number of applications. ${ }^{(8)}$ Furthermore, cryoablation in the experimental setting is associated with less tissue architecture disruption and could reduce complications such as pulmonary venous stenosis and atrio-oesphageal fistula. ${ }^{(9,10)}$ Cryoballoon ablation has been demonstrated to have efficacy and safety profile comparable to RF ablation. ${ }^{(11)}$ A recent meta-analysis of 16 clinical trials comparing cryoablation with $\mathrm{RF}$ ablation concluded that cryoballoon ablation (CBA) was non-inferior to radiofrequency ablation (RFA) for paroxysmal AF. RF ablation was associated with a higher groin complications and pericardial effusion/cardiac tamponade, whereas CBA was associated with higher rates of transient and persistent phrenic nerve injury. ${ }^{(12)}$

Acute procedural success after AF ablation does not necessarily translate into long term arrhythmia-free survival, which is lower in persistent than paroxysmal AF. Moreover, AF ablation procedures are not free of complications. Therefore the need for proper risk assessment to predict recurrence after ablation is of paramount importance. There are many studies which have explored the predictors of recurrence after the index AF ablation. ${ }^{(13-16)}$ In the present study, we retrospectively assessed the value of several imaging and clinical tools to predict 1-year recurrence of $\mathrm{AF}$ after balloon cryoablation.

\section{Materials and Methods:-}

Seventy patients who underwent their first cryoballoon ablation of pulmonary veins for symptomatic drug-refractory atrial fibrillation (AF) in electrophysiology laboratory, Spedali Civili Di Brescia, Italy, in the period between January 2013 and November 2015 , with available cardiac MRI study done before the ablation procedure, had been divided into two groups: group A included patients who developed AF recurrence at one year post-ablation, while group B included patients who remained arrhythmia-free.

\section{Pre-procedural Preparation:-}

For each patient, the $\mathrm{CHA}_{2} \mathrm{DS}_{2}-\mathrm{VASc}$ was calculated. To rule out the presence of left atrial (LA) and LAA thrombi, all patients underwent two dimensional (2D) transoesophageal echocardiography (TEE) the day before the procedure, along with a transthoracic echocardiogram for the assessment of LA dimension, left ventricular ejection fraction (LVEF), and valvular function. Class IC antiarrhythmic drugs were discontinued prior to ablation. Procedures were performed either with continued oral anticoagulation using warfarin and therapeutic INR (2.0 to 3.0) or NOACs. Patients with left atrial diameter $>55 \mathrm{~mm}$ were excluded.

\section{Pulmonary vein isolation procedure:-}

Briefly, all procedures were carried out in conscious sedation. A deflectable decapolar catheter was inserted through right femoral vein and positioned into the coronary sinus to guide the transseptal puncture and was subsequently moved to the superior vena cava to stimulate the right phrenic nerve during treatment of the right PVs. A single transseptal puncture was performed using a needle system (BRK, St. Jude Medical, St. Paul, MN, USA) and a standard transseptal sheath (SL0 8F or 8.5F, St. Jude Medical, St. Paul, MN, USA), subsequently exchanged with a steerable 15F sheath (FlexCath Advance ${ }^{\circledR}, 15 \mathrm{~F}$, Medtronic ${ }^{\odot}$, Inc., Minneapolis, MN, USA). Before transseptal puncture, heparin was administered intravenously as bolus $(10000 \mathrm{U})$ followed by a continuous infusion $(1000 \mathrm{U} / \mathrm{hr})$ to obtain ACT level > $350 \mathrm{sec}$. The FlexCath was continuously irrigated with heparinized saline $(2 \mathrm{~mL} / \mathrm{hr})$. In cases of difficulty to achieve a complete venous occlusion due to the persistence of leak at the inferior portion of the PV's ostium, a "pull down" maneuver as well as other changes of the balloon orientation were attempted. ${ }^{(17)}$ After treatment of all PVs, bidirectional block was confirmed with pacing maneuvers through the Achieve ${ }^{\circledR}$ mapping catheter (Medtronic ${ }^{\circ}$, Inc., Minneapolis, MN, USA) and the distal poles of the coronary sinus catheter. Any residual conduction into the PVs was treated by further cryotherapy applications. Successful PVI was confirmed when all PV potentials were abolished or were dissociated at least 20 minutes after the last cryotherapy application to that vein.

\section{Post-procedural care and Management:-}

Transthoracic echo was done immediately after the procedure to rule out pericardial effusion. The patients were then monitored by continuous ECG recording in the following 48 hours. Anticoagulation (whether VKA or NOACs) was restarted the evening of the same day and continued for at least 3 months. The patients were discharged 48 hours 
post procedure provided that a pre-discharge echocardiogram ruled out pericardial effusion. Antiarrhythmic drugs were administered throughout the blanking period (first 3 months); afterwards the therapy could be stopped or continued according to the follow up and preference of the referring electrophysiologist. The follow up data are obtained from the records at 3 months and one year post-procedure. Some patients, according to a clinical indication, received implantable loop recorder. Patients were asked to record their 12-lead electrocardiogram whenever they experienced symptoms suggestive of AF.

The clinical data and echo measurements (including LA diameter and left ventricular ejection fraction) were collected form the patient records. All patients were assessed by pre-procedural Contrast enhanced MRI (CMR was performed on a 1.5 Tesla Philips Achieva ${ }^{\circledR}$ MRI scanner (Amsterdam, Netherlands) using a five-element phasedarray cardiac coil) to obtain the following informations:

1. PV drainage pattern: A common PV ostium was deemed to be present if the superior and inferior veins coalesce prior to the inflection point at which the PV ostia meet the left atrial wall, thereby forming a single atrio-pulmonary venous junction ${ }^{(18,19)}$, An accessory or supernumerary PV was identified by the presence of a vein other than the superior or inferior lobe veins entering the atrium via a distinct ostium. ${ }^{(20)}$

2. PV ostial size and shape (Figure 1 and 2): To describe the shape we calculated the ovality index of every individual pulmonary vein at the ostial level: Ovality means to what extent the ostial shape deviated from the perfect circularity.

Ovality or non-circularity index $=2 \times(a-b) /(a+b)^{(24)}$

Where $a$ is the length of the major axis and $b$ is the length of the minor axis.

The follow up of the patients at 3 months and 1 year post-procedure were collected form the records. Early recurrence of $\mathrm{AF}$ will be defined as detection of $\mathrm{AF}$ (at least $30 \mathrm{~s}$ duration when assessed with ECG monitoring) within 3 months of ablation. One year recurrence of AF will be defined as detection of AF (at least $30 \mathrm{~s}$ duration when assessed with ECG monitoring) at 1 year following AF ablation .

\section{Statistical Analysis:-}

Statistical analysis was done using IBM SPSS statistics program version 21 and Medcalc programs. Quantitative data were described by mean and median as measures of central tendency\& standard deviation, minimum and maximum as measures of dispersion, while categorical variables were summarized by frequency and percent. A Pvalue of $<0.05$ was considered statistically significant. Multivariate stepwise logistic regression using backward (Wald and Likelihood ratio) methods was done for statistically significant risk factors by bivariate analysis.

\section{Results:-}

Of the total of 70 patients who were included in the present analysis, Fourteen (20\%) patients of the whole study population developed AF recurrence (group A) versus Fifty-six (80\%) who remained free from arrhythmia recurrence at one year (group B). Table 1 describes the clinical and demographic data of both study groups. Compared to group $\mathrm{B}$, the patient in group A were significantly older $(\mathrm{p}=0.05)$, with more arterial hypertension $(p=0.03)$, persistent atrial fibrillation $(p=0.05)$ and early recurrence of atrial fibrillation $(p=0.001)$.

\section{Echocardiography parameters:-}

Left atrial diameter; the mean left atrial anteroposterior diameter was $47.8 \pm 6.6 \mathrm{~mm}$ and $40.5 \pm 5.6 \mathrm{~mm}$ in group A and $\mathrm{B}$ respectively $(\mathrm{p}=0.001)$. Left ventricular ejection fraction; the mean left ventricular ejection fraction measured by $\mathrm{M}$ Mode was $57.7 \pm 5.5$ in group $A$ and $57.3 \pm 3.2$ in group $\mathrm{B}(\mathrm{P}=0.83)$.

\section{MRI assessment of pulmonary vein drainage pattern:-}

There was no difference between group A and group B in the presence of common left or right pulmonary veins as well as supernumerary right pulmonary veins (Table 2 ).

\section{Pulmonary vein (PV) ostial ovality index:-}

After exclusion of the patients with common pulmonary veins; The ostial ovality index, assessed at each individual pulmonary vein ostium, was not significantly different between both groups (Table 3 ). The mean ovality index of the left pulmonary veins $(0.41 \pm 0.15)$ was significantly higher than the mean ovality index of right pulmonary veins $(0.21 \pm 0.09)$, $\mathrm{p}=0.001$. So, left pulmonary veins were more oval than right pulmonary veins. 
A multivariate analysis using logistic regression model was done with the following variables which are statistically significant by univarite analysis: Hypertension; Age; Left atrial diameter; Persistent AF and ERAF. According to wald statistics, the most significant predictor for recurrence is age. The odds of recurrence increases by $20 \%$ (OR 1.19; $95 \%$ CI: 1.06 to 1.34 ) per one year increase in age. One unit increase in left atrial diameter increases the chance of recurrence by $13 \%$ (OR: .87; $95 \%$ CI .768 to .985).

Figure 1:- shows the manual alignment of both longitudinal and transverse axes of the right superior pulmonary vein (RSPV) at the ostial level. So index of ovality at the PV ostial level $=2 \times$ (maximal diameter- minimal diameter) / (maximal diameter + minimal diameter).

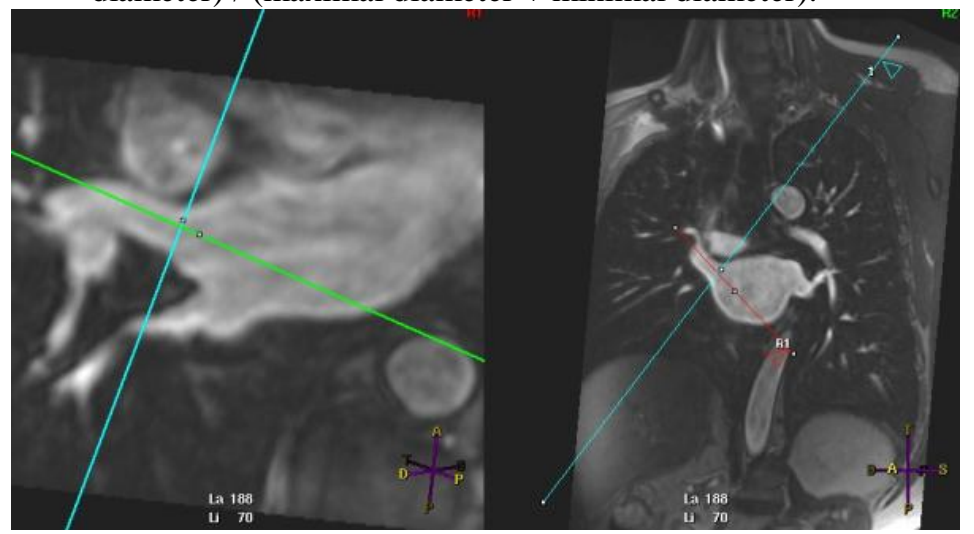

Figure 2:- short axis view at the level of RIPV ostium shows the manual measurements of maximal diameter, minimal diameter in $\mathrm{mm}$ and ostial area in $\mathrm{mm}^{2}$.

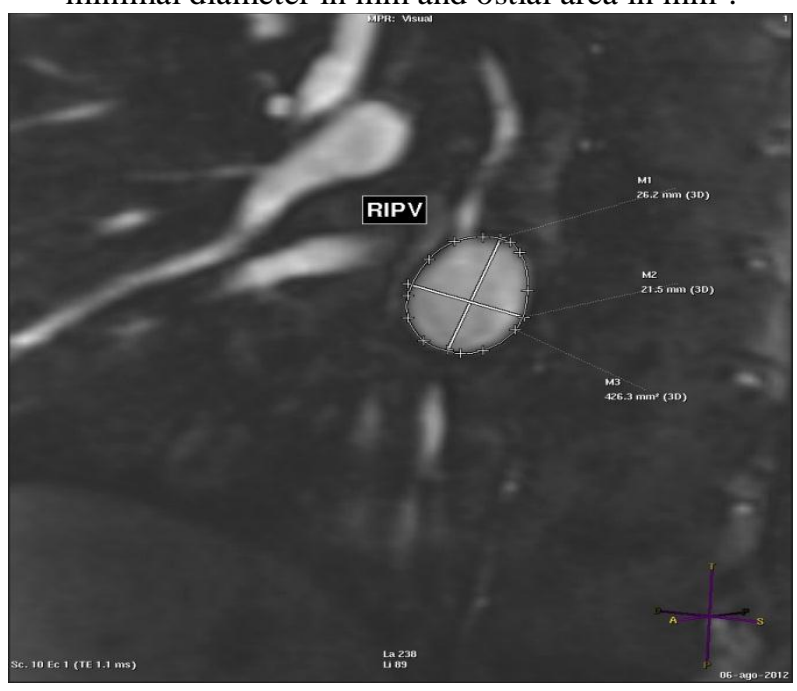

Table 1:- Comparison between both study groups concerning the clinical variables

\begin{tabular}{|c|c|c|c|c|c|}
\hline & \multicolumn{2}{|c|}{$\begin{array}{l}\text { Group A } \\
(\mathrm{n}=14)\end{array}$} & \multicolumn{2}{|c|}{$\begin{array}{l}\text { Group B } \\
(n=56)\end{array}$} & \multirow[t]{2}{*}{$\mathrm{P}$} \\
\hline & No. & $\%$ & No. & $\%$ & \\
\hline \multicolumn{6}{|l|}{ Gender } \\
\hline Male & 9 & 64.3 & 50 & 71.4 & \multirow{2}{*}{0.52} \\
\hline Female & 5 & 35.0 & 20 & 28.0 & \\
\hline \multicolumn{6}{|l|}{ Age (years) } \\
\hline Min. - Max. & \multicolumn{2}{|c|}{$60.0-80.0$} & \multicolumn{2}{|c|}{$35.0-76.0$} & \multirow[t]{3}{*}{0.05} \\
\hline Mean \pm SD. & \multicolumn{2}{|c|}{$66.86 \pm 10.64$} & \multicolumn{2}{|c|}{$55.16 \pm 10.38$} & \\
\hline Median & \multicolumn{2}{|c|}{66.0} & \multicolumn{2}{|c|}{52.50} & \\
\hline Diabetes & 3 & 25.0 & 10 & 27.0 & 1.000 \\
\hline
\end{tabular}




\begin{tabular}{|l|l|l|l|l|l|}
\hline Hypertension & 10 & 71.4 & 16 & 28.6 & $\mathbf{0 . 0 3}$ \\
\hline CAD & 1 & 25.0 & 3 & 75.0 & 1.000 \\
\hline Heart failure & 5 & 35.7 & 18 & 32.1 & 1.000 \\
\hline Renal dysfunction & 0 & 0.0 & 1 & 1.8 & 1.000 \\
\hline History of atrial flutter & 3 & 21.4 & 10 & 17.9 & 0.71 \\
\hline Persistent AF & 7 & 50.0 & 11 & 19.6 & $\mathbf{0 . 0 5}$ \\
\hline ERAF & 8 & 57.1 & 7 & 12.5 & $\mathbf{0 . 0 0 1}$ \\
\hline
\end{tabular}

CAD, coronary artery disease ; AF, atrial fibrillation; ERAF, early recurrence of atrial fibrillation

Table 2:- Comparison between the two study groups according to drainage pattern of the pulmonary veins in the left atrium

\begin{tabular}{|c|c|c|c|c|c|}
\hline & \multicolumn{2}{|c|}{$\begin{array}{l}\text { Group A } \\
(n=14)\end{array}$} & \multicolumn{2}{|c|}{$\begin{array}{l}\text { Group B } \\
(n=56)\end{array}$} & \multirow[t]{2}{*}{$\mathrm{p}$} \\
\hline & No. & $\%$ & No. & $\%$ & \\
\hline \multicolumn{6}{|l|}{ Common right PV } \\
\hline & 2 & $14 \%$ & 0 & 0.0 & 0.12 \\
\hline Common left PV & 4 & 28.0 & 6 & 24.0 & 0.72 \\
\hline Supernumerary right $\mathrm{PV}$ & 3 & 21.0 & 5 & 20.0 & 0.10 \\
\hline
\end{tabular}

PV, pulmonary vein.

Table 3:- Comparison of both study groups regarding the mean ovality index of each individual pulmonary vein.

\begin{tabular}{|l|l|l|l|}
\hline & Mean ovality index of Group A & Mean ovality index of Group B & P value \\
\hline LSPV & $0.41 \pm 0.22$ & $0.33 \pm 0.14$ & 0.26 \\
\hline LIPV & $0.471 \pm 0.22$ & $0.46 \pm 0.19$ & 0.89 \\
\hline RSPV & $0.16 \pm 0.14$ & $0.24 \pm 0.13$ & 0.09 \\
\hline RIPV & $0.16 \pm 0.10$ & $0.24 \pm 0.08$ & 0.06 \\
\hline
\end{tabular}

LSPV, left superior pulmonary vein, LIPV, left inferior pulmonary vein, RSPV, right superior pulmonary vein, RIPV, right inferior pulmonary vein.

Figure 3:- Shows the difference in the mean ovality index between right and left pulmonary veins in both groups.

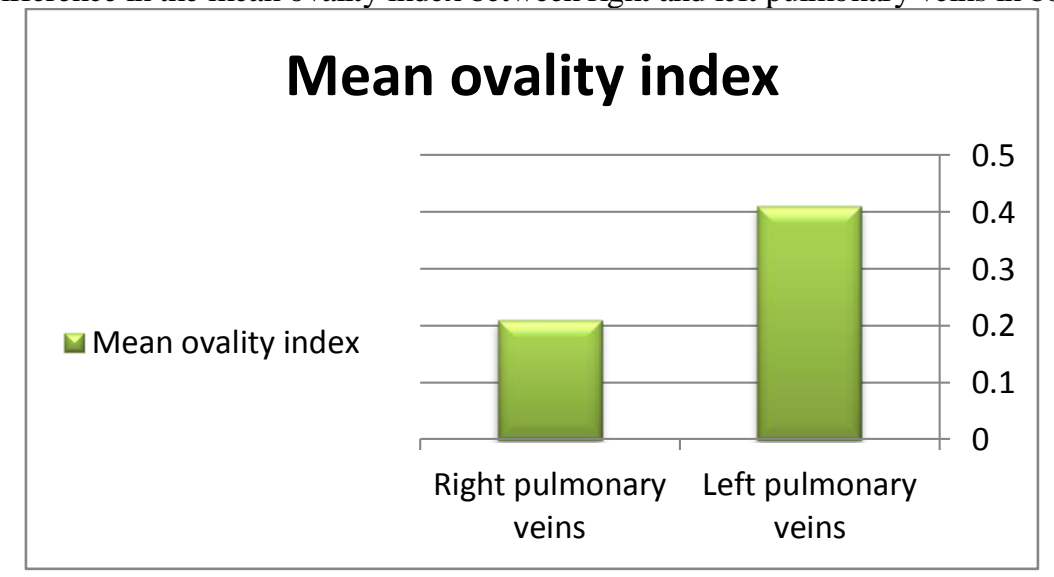

\section{Discussion:-}

Efficacy and safety of pulmonary vein isolation by means of balloon-based cryoablation has been established by several observational studies. ${ }^{(21)}$ In a systematic review of 23 studies, most of them including only patients with paroxysmal AF, Andrade et al. ${ }^{(22)}$ reported a successful acute PVI in more than $98 \%$ of the patients, with $73 \%$ of paroxysmal and $45.1 \%$ of persistent AF patients having 1 year freedom form AF recurrence out of the blanking period. Despite the constant improvement in the procedural efficacy and safety with cryoballoon ablation, a certain failure rate still remains, and this could be partially attributed to patient selection. As such, the preoperative estimation of the recurrence probability may aid in the patient selection process, particularly considering the 
heterogeneous follow up modalities, leading to asymptomatic AF episode missing, and the highly variable use and definition of predictors for AF recurrence.

In this study, we investigated the predictors of AF recurrence in patients undergoing cryoballoon ablation for paroxysmal or persistent AF. The main findings were as follows: clinical success in regard to the freedom from recurrent AF was 80\%. Patients who experienced AF recurrence at one year (Group A) were older, with more frequent hypertension, persistent AF, early recurrence of atrial fibrillation and larger left atrial diameter. Other comorbidities (diabetes mellitus, coronary artery disease, heart failure, renal dysfunction), history of atrial flutter, atypical pulmonary vein drainage pattern and ostial ovality index, did not predict arrhythmia recurrence at one year. Multivariate analysis of our five significant variables showed that age and left atrial diameter were the two independent predictors for AF recurrence after cryoablation.

Khoueiry $\mathrm{Z}$ et al ${ }^{(23)}$, evaluated the mid-term outcomes of cryoballoon ablation in an unselected population with paroxysmal AF who underwent a first procedure of cryoballoon ablation or radiofrequency from a pulmonary vein anatomical viewpoint. Comparisons between both techniques were performed regarding safety and efficacy endpoints, according to pulmonary vein (PV) anatomical variants. They found no difference in the incidence of relapse $(17.0 \%$ cryoballoon ablation vs. $14.1 \%$ radiofrequency, $\mathrm{P}=0.25)$. Moreover, no interaction of $\mathrm{PV}$ anatomical variants on mid-term procedural success. In the present study, we observed no difference between both groups regarding the presence of common left pulmonary venous ostium, common right ostium or accessory right pulmonary vein.

Schmidt $\mathrm{M}$ et al (24), investigated the role of Ovality index of each individual pulmonary vein ostium on PV occlusion grading and AF recurrence rate during PVI with cryoballoons (both 23 and 28 mm balloons). They used cardiac computed tomography prior to procedure to assess PV ostial diameter in 168 consecutive AF patients. The success rate at $12 \pm 6$ months follow-up was 69\%. They observed that , left-sided PVs were significantly "more oval" than right sided PVs. Complete PV occlusion was more often achieved in patients with a "less oval“" PV ostial anatomy and corresponded to a lower AF recurrence rate at long term follow-up. Furthermore, ostial PV anatomy had an important impact on clinical outcome; Patients with AF recurrence had "more oval" left-sided PVs compared to patients free from AF recurrence, whereas no significant association was found for right sided PVs. In the present study, the left pulmonary veins were found to be more oval than the right veins. We did not observe statstical significant relationship between 1-year recurrence of AF after cryoablation and the mean ovality index of every individual pulmonary vein.

Many studies also show that increased left atrial diameter (LAD) is an important predictor of the recurrence of AF after pulmonary vein isolation. Ejima K et al. ${ }^{(25)}$ followed 80 patients with non-paroxysmal AF refractory to antiarrhythmic drugs after PVI For about 3 years. They observed that left atrial volume (LAV) was the only independent predictor of atrial tachyarrhythmia recurrences not only after single procedures, but also after the final procedures. Moreover, Repeat ablation procedures increased the best cut-off value for predicting recurrences. In the present study, the mean left atrial diameter was higher in patients who developed AF recurrence than in patients who remained free of recurrence at 1-year follow-up post procedure.

Age was proved to be a significant predictor for AF recurrence after catheter ablation. Lee SH et al ${ }^{(26)}$ investigated the factors affecting the rhythm outcomes after the maze procedure in patients with a giant LA and AF-associated mitral valve disease to determine the best candidates for the maze procedure in this population. The 5-year AF-free rate was $87.1 \% \pm 4.3 \%$ in patients 50 years or younger and $77.3 \% \pm 4.1 \%$ in patients older than 50 years $(\mathrm{P}=.001)$. On multivariate analysis, advanced patient age was the only significant, independent risk factor for AF recurrence. In our study, age was an independent predictor, on multivariate analysis, of arrhythmia recurrence after cryoablation. The odds of recurrence increases by 20\% (OR 1.19; 95\% CI: 1.06 to 1.34) per one year increase in age.

Early recurrence after catheter ablation of AF is fairly common: Bertaglia found $46 \%$ of atrial tachy-arrhythmias relapse during the first three months of follow-up. ${ }^{(27)}$ Moreover the incidence of ER is maximum soon after the procedure, which decreases in the following days. ${ }^{(28)}$ Although, the prevalence of ER is not negligible, it has been widely recognized that a good proportion of patients experiencing ER are free of significant atrial arrhythmias at prolonged observation. Nevertheless, the occurrence of LR is more frequent in the subgroup with ER. ${ }^{(29)}$ Similar findings were reported by Themistoclakis and coworkers ${ }^{(30)}$ They investigated the predictors of AF recurrence and 
the relationship between early recurrence (EAT) and late recurrence (LAT) after AF ablation in 1298 mixed AF patients. After a single ablation procedure, EAT developed in 514 (40\%) patients and LAT in 292 (22\%) patients. EAT was Independent predictor of LAT. The risk of LAT was inversely related to the time to first EAT occurrence (LAT occurred more frequently in patients who experienced the first EAT in the third month than in those who experienced the first EAT in the second or first month (98\% vs $69 \%$ and $44 \%$,). Notably, $49 \%$ of patients with EAT did not experience LAT. Moreover, ERAF was the most important predictor for late AF recurrence in a study ${ }^{(29)}$ of 61 paroxysmal AF patients who underwent balloon cryoablation.

Canpolat U et al. ${ }^{(31)}$ proposed a new scoring system in the prediction of recurrence after AF ablation with cryoballoon in 236 patients. According to univariate Cox proportional hazard regression analysis, hypertension, BMI, current smoking, left atrium diameter, nonparoxysmal AF type, duration of AF history and early recurrence were significantly associated with AF recurrence ( $\mathrm{p}<0.05)$.Concordant with the previous results, patients who developed recurrence of AF at 1 year after cryoballoon ablation, in the present study, had more hypertension and persistent AF.

Proper patient selection could avoid unnecessary procedures and so the expenditure, especially in the light of the currently increased procedure costs, and could prevent potential ablation-related complications. Moreover, the decision to refer a patient with AF for catheter ablation should balance the indication with the procedure outcomes. Therefore, proper assessment of the procedure risks including the recurrence risk could be useful in daily practice, facilitating the decision making process of the AF patients and can be a part of the discussion with the patient. With a low risk profile for recurrence i.e. young patient with paroxysmal AF, no or minimal structural heart disease and refractory symptoms, physicians may be more enthusiastic to early patient referral to ablation by virtue of low anticipated arrhythmia recurrence and better long-term outcomes. In asymptomatic patient, ablation is not indicated even with a very low risk for recurrence. On the other hand, patients with a high risk profile, i.e. old patient with persistent AF, hypertension and significant structural hear disease should be thoroughly informed about the high risk of recurrence and the importance of maintaining regular follow up and monitoring after ablation. However, in such type of patients, ablation may be decided despite anticipated high recurrence risk in the presence of refractory symptoms. Furthermore, in a patient with a high risk of recurrence, the decision may be taken to avoid redo-ablation considering the increased risk of further recurrences.

\section{Conclusions:-}

Age and left atrial diameter are independent predictors for AF recurrence after cryoballoon-guided pulmonary vein isolation. We highlight the importance of proper assessment of the risk of arrhythmia recurrence before referring the patient for ablation.

\section{Conflict of Interest:-}

Mohamed Sanhoury received research grant form Alexandria University, Egypt.

Antonio Curnis is a consultant to Boston Scientific, Biotronik, Medtronic, St Jude Medical, and Sorin Group.

\section{References:-}

1. Wolf PA, Benjamin EJ, Belanger AJ, et al. Secular trends in the prevalence of atrial fibrillation: the Framingham Study. Am Heart J 1996;131:790-5.

2. Camm AJ, Kirchhof P, Lip GY, et al. Guidelines for the management of atrial fibrillation: the Task Force for the Management of Atrial Fibrillation of the European Society of Cardiology (ESC). Eur Heart J 2010;31:2369429.

3. Go AS, Hylek EM, Phillips KA, et al. Prevalence of diagnosed atrial fibrillation in adults: national implications for rhythm management and stroke prevention: the AnTicoagulation and Risk Factors in Atrial Fibrillation (ATRIA) Study. JAMA 2001;285: 2370-5.

4. Haïssaguerre M, Marcus F I, Fischer B, et al. Radiofrequency catheter ablation in unusual mechanisms of atrial fibrillation: report of three cases. J. Cardiovasc. Electrophysiol. 1994 Sep;5 (9):743-51.

5. Kojodjojo P, O'Neill MD, Lim PB, Malcolm-Lawes L, Whinnett ZI, Salukhe TV, et al. Pulmonary venous isolation by antral ablation with a large cryoballoon for treatment of paroxysmal and persistent atrial fibrillation: medium-term outcome and non-randomised comparison with pulmonary venous isolation by radiofrequency ablation. Heart 2010; 96(17):1379-84. 
6. Haissaguerre M, Jais P, Shah DC, Takahashi A, Hocini M, Quiniou G, et al. Spontaneous initiation of atrial fibrillation by ectopic beats originating in the pulmonary veins. N Engl J Med 1998;339(10):659-66.

7. Kettering K, Greil GF, Fenchel M, Kramer U, Weig HJ, Busch M, et al. (2009).Catheter ablation of atrial fibrillation using the Navx/Ensite-system and a CT-/MRI-guided approach. Clin Res Cardiol. 2009; 98(5):28596.

8. Evranos B, Aytemir K, Oto A, Okutucu S, Karakulak U, Levent Şahiner, et al. Predictors of atrial fibrillation recurrence after atrial fibrillation ablation with cryoballoon. Cardiol J 2013;20(3):294-303.

9. Khairy P, Dubuc M. Transcatheter cryoablation part I: preclinical experience. Pacing Clin Electrophysiol 2008;31(1):112-20.

10. Lemola K, Dubuc M, Khairy P. Transcatheter cryoablation part II: clinical utility. Pacing Clin Electrophysiol 2008;31(2):235-44.

11. Kuck KH, Brugada J, Fürnkranz A, Metzner A, Ouyang F, Chun K.R, et al. Cryoballoon or Radiofrequency Ablation for Paroxysmal Atrial Fibrillation (FIRE AND ICE trial). N Engl J Med 2016;374(23):2235-45.

12. Garg J, Chaudhary R, Palaniswamy C, Shah N, Krishnamoorthy P, Bozorgnia B ,et al. Cryoballoon versus Radiofrequency Ablation for Atrial Fibrillation: A Meta-analysis of 16 Clinical Trials. J Atr Fibrillation. OctNov 2016; 9(3):24-32.

13. Tang RB, Dong JZ, Liu XP, Kang JP, Ding SF, Wang L et al. Obstructive sleep apnoea risk profile and the risk of recurrence of atrial fibrillation after catheter ablation. Europace 2009;11(1):100-5.

14. Berruezo A, Tamborero D, Mont L, Benito B, Tolosana JM, Sitges M et al. Preprocedural predictors of atrial fibrillation recurrence after circumferential pulmonary vein ablation. Eur Heart J 2007;28(7):836-41.

15. D'Ascenzo F, Corleto A, Biondi-Zoccai G, Anselmino M, Ferraris F, di Biase L et al. Which are the most reliable predictors of recurrence of atrial fibrillation after transcatheter ablation? A meta-analysis. Int J Cardiol 2013;167(5):1984-9.

16. Zhuang J, Wang $\mathrm{Y}$, Tang $\mathrm{K}$, Li X, Peng W, Liang $\mathrm{C}$ et al. Association between left atrial size and atrial fibrillation recurrence after single circumferential pulmonary vein isolation: a systematic review and metaanalysis of observational studies. Europace 2012; 14(5):638-45.

17. Ahmed H, Neuzil P, Skoda J, D'Avila A, Donaldson DM, Laragy MC, et al. The permanency of pulmonary vein isolation using a cryoballoon ablation catheter. J Cardiovasc Electrophysiol 2010; 21(7):731-7.

18. McLellan A J, Ling LH, Ruggiero D, et al. Pulmonary vein isolation: the impact of pulmonary venous anatomy on long-term outcome of catheter ablation for paroxysmal atrial fibrillation. Heart Rhythm. 2014 Apr;11(4):549-56.

19. Van der Voort PH, van den Bosch H, Post JC, et al. Determination of the spatial orientation and shape of pulmonary vein ostia by contrast-enhanced magnetic resonance angiography. Europace 2006;1:1-6.

20. Thorning C, Hamady M, Ping Liaw JV, et al. CT evaluation of pulmonary venous anatomy variation in patients undergoing catheter ablation for atrial fibrillation. Clin Imaging 2011;35(1):1-9.

21. Packer DL, Kowal RC, Wheelan KR, Irwin JM, Champagne J, Guerra PG, et al. Cryoballoon ablation of pulmonary veins for paroxysmal atrial fibrillation: first results of the North American Arctic Front (STOP AF) pivotal trial. J Am Coll Cardiol 2013; 61(16):1713-23.

22. Andrade JG, Khairy P, Guerra PG, Deyell MW, Rivard L, Macle L, et al. Efficacy and safety of cryoballoon ablation for atrial fibrillation: a systematic review of published studies. Heart Rhythm 2011; 8(9):1444-51.

23. Khoueiry Z, Albenque JP, Providencia R, et al. Outcomes after cryoablation vs. radiofrequency in patients with paroxysmal atrial fibrillation: impact of pulmonary veins anatomy. Europace. 2016 Sep;18(9):1343-51.

24. Schmidt M, Dorwarth U, Straube F, et al . Cryoballoon in AF ablation: Impact of PV ovality on AF recurrence. Int J Cardiol. 2013 Jul 15;167(1):114-20.

25. Ejima K, Arai K, Suzuki T, et al. Long-term outcome and preprocedural predictors of atrial tachyarrhythmia recurrence following pulmonary vein antrum isolation based catheter ablation in patients with non paroxysmal atrialfibrillation. J Cardiol. 2014 Jul;64(1):57-63.

26. Lee SH, Kim JB, Cho WC, al. The influence of age on atrial fibrillation recurrence after the maze procedure in patients with giant left atrium.J Thorac Cardiovasc Surg. 2011 Apr;141(4):1015-9.

27. Bertaglia E, Stabile G, Senatore G, et al. Predictive value of early atrial tachyarrhythmias recurrence after circumferential anatomical pulmonary vein ablation. Pacing Clin Electrophysiol. 2005;28:366-371. 7.

28. Joshi S, Choi AD, Kamath GS et al. Prevalence, predictors, and prognosis of atrial fibrillation early after pulmonary vein isolation: findings from 3 months of continuous automatic ECG loop recordings. J Cardiovasc Electrophysiol, 2009; 20: 1089-1094. 
29. Andrade JG, Khairy P, Verma A, et al. Early recurrence of atrial tachyarrhythmias following radiofrequency catheter ablation of atrial fibrillation. Pacing Clin Electrophysiol. 2012;35:106-16.

30. Themistoclakis S, Schweikert RA, Saliba $\quad$ WI, et al. Clinical predictors and relationship between early and late atrial tachyarrhythmias afterpulmonary vein antrum i solation. Heart Rhythm. 2008 May;5(5):679-85.

31. Canpolat U, Aytemir K, Yorgun H, et al. A proposal for a new scoring system in the prediction of catheter ablation outcomes: Promising results from the Turkish Cryoablation Registry. Int J Cardiol 2013;169(3):201-6. 